\title{
Protecting-Group-Free Synthesis of Glycomonomers and Glycopolymers from Free Saccharides
}

\author{
Tomonari Tanaka \\ Department of Biobased Materials Science, Graduate School of Science and Technology, Kyoto Institute of Technology, \\ Matsugasaki, Sakyo-ku, Kyoto 606-8585, Japan \\ FAX: +81-75-724-7547, E-mail: t-tanaka@kit.ac.jp
}

(Received on November 18, 2015, accepted on January 22, 2016)

Key Words: glycomonomer, oligosaccharide, direct activation, glycopolymer, cluster effect

\begin{abstract}
Glycopolymers are synthetic polymers with pendant saccharides developed as glycocluster molecules incorporating multivalent forms of saccharides. Although many different glyco-monomers and -polymers have been described, most reported synthetic methods require multiple laborious steps such as the protection and deprotection of hydroxy groups on the saccharide, making these synthetic approaches difficult to apply to oligosaccharides with higher molecular weight. In contrast, there have been few reports of the synthesis of glyco-monomers and -polymers from free saccharides not requiring protection of the saccharide hydroxy groups. This review describes the protecting-group-free syntheses by our group and others of glyco-monomers and -polymers, and reviews the results of binding assays using our glycopolymers with lectins and influenza viruses.
\end{abstract}

\section{A. Introduction}

Glycopolymers comprise a synthetic polymer with pendant saccharides and have received much attention from scientists working in diverse fields such as polymer chemistry, materials science, and biochemistry (1-6). Although saccharideprotein interactions are generally weak, these interactions are amplified by multivalent forms of saccharides. Such multivalent saccharides give rise to the "glycocluster effect" $(7,8)$ underlying various biological processes. Glycopolymers similarly give rise to artificial glycoclusters. These synthetic glycoclusters amplify the saccharide signals of natural ligands, glycodendrimers (9-11), glyconanoparticles (12-15), glycosylated surfaces and scaffolds, and also conjugate with DNA, liposomes, and nanotubes (16-19).

The synthesis of glycopolymers is laborious and requires multistep processes, including the synthesis of glycomonomers, the synthesis of saccharide derivatives with functional groups from free saccharides, and the protection and deprotection of hydroxy, carboxy, and amino groups on the saccharide moieties. Typical routes to glycopolymers consist of both synthesizing glycomonomers with a polymerizable group and post-attaching of glycosyl derivatives to the polymer backbone. Clearly, a simpler and more efficient synthetic method applicable to oligosaccharides is desirable for the preparation of glycopolymers from free saccharides. In this review, I first introduce previous studies describing the synthesis of glyco-monomers and -polymers from free saccharides without the use of any protecting group. Then, I focus on our recent approach for synthesizing glyco-monomers and -polymers from free saccharides, and describe the results of binding assays of these glycosylated compounds with lectins and influenza viruses.

\section{B. Previous Methods of Synthesizing Glycomonomers and Glycopolymers}

Many synthetic methods and types of glyco-monomers and -polymers have been reported to date. A typical approach for the synthesis of glycopolymers involves the polymerization of glycomonomers (Fig. 1A). Another approach involves attaching saccharide moieties to a polymer backbone (Fig. 1B). Both approaches, however, involve the laborious synthesis of glycosyl derivatives from free saccharides. In the former approach, glycomonomers are synthesized by first protecting all the hydroxy groups on the starting free saccharides, then activating their anomeric position, introducing a polymerizable group at the anomeric position, and finally deprotection (Fig. 1Aa $\rightarrow$ b). The resulting glycomonomers are subjected to polymerization reaction to obtain glycopolymers (Fig. 1Ac). The deprotection step is often performed after the polymerization reaction (Fig. $1 \mathrm{Aa} \rightarrow \mathrm{d} \rightarrow \mathrm{e}$ ). In the later approach, glycopolymers are synthesized through a post-attaching reaction with glycosyl derivatives possessing an attachable group such as azide, acetylene, thiol, or other easily reactive group (Fig. $1 \mathrm{Bf} \rightarrow \mathrm{g} \rightarrow \mathrm{h}$ ). This procedure necessitates not only the multi-step synthesis of a glycosyl derivative, but also the preparation of a polymer backbone decorated with pendant functional groups for attaching saccharide moieties. The deprotection step is conducted following attachment of the saccharide moieties to the polymer backbone, as in the first method (Fig. 1Bf $\rightarrow \mathrm{i} \rightarrow \mathrm{j}$ ).

Little has been reported regarding the protecting-group-free synthesis of glycomonomers from free saccharides. The Fischer glycosylation method directly provided a glycomonomer from glucose by using an excess of hydroxyethyl acrylate in the pres- 

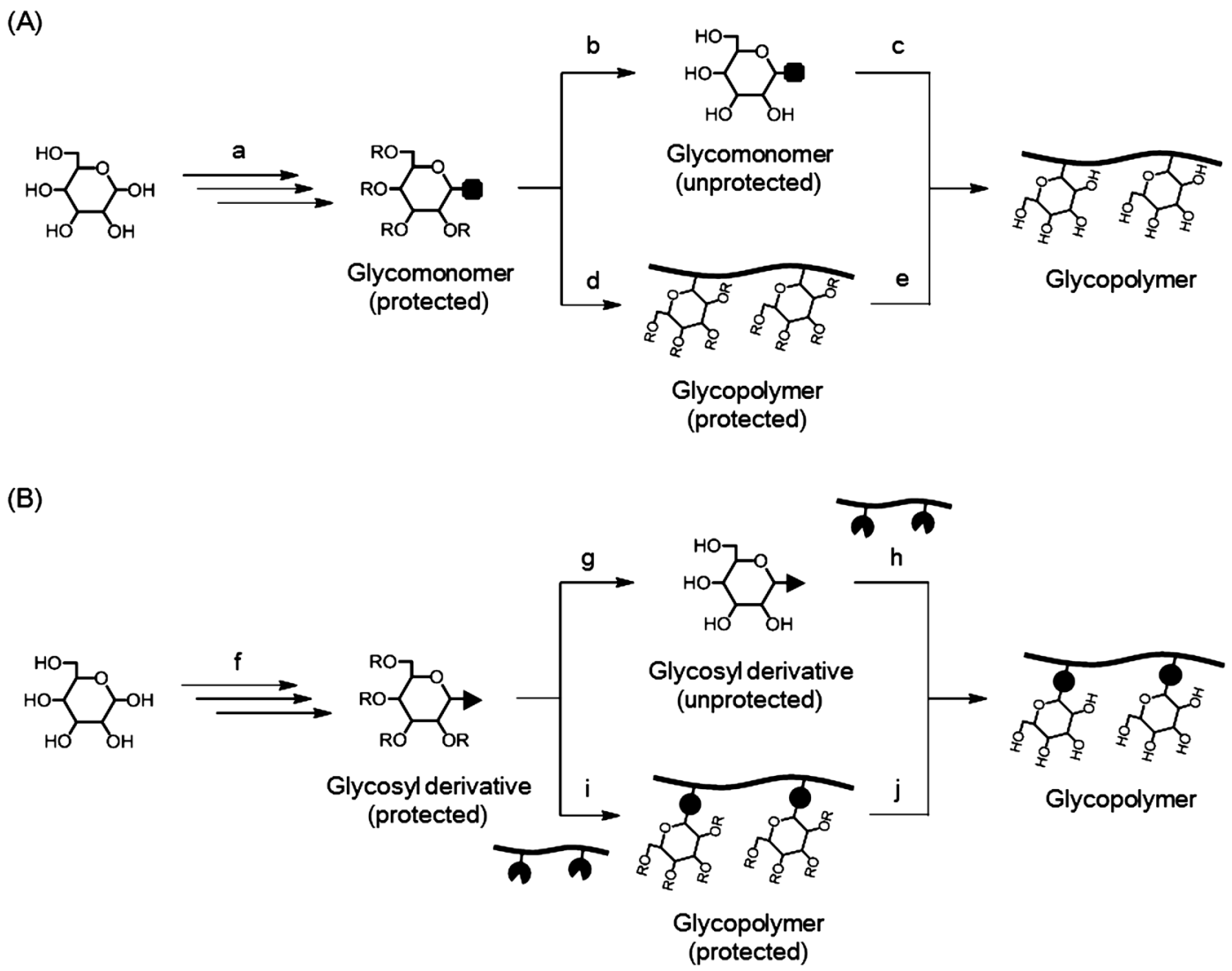

Fig. 1. Strategies for synthesizing glycopolymers from free saccharides. (A) Methods for synthesizing glycomonomers, and subsequent polymerization. (a) Synthesis of protected glycomonomer, including protection of the hydroxy groups and introduction of the polymerizable group at the anomeric position, (b, e) deprotection, (c, d) polymerization. (B) Methods for post-attaching saccharide to the polymer backbone. (f) Synthesis of protected glycosyl derivative, including protection of the hydroxy groups and introduction of a functional group at the anomeric position, (g, $\mathrm{j}$ ) deprotection, (h, i) attaching saccharide moieties to the polymer backbone.

ence of phosphomolybdic acid as a catalyst (Fig. 2A). However, this method cannot be applied to oligosaccharides and to control stereoselectivity at the anomeric position (20). Another approach, involving the synthesis of lactone derivatives, is well known (Fig. 2B) $(21,22)$. For example, Kobayashi et al. synthesized styrene derivatives possessing di- and trisaccharides by coupling vinylfunctionalized amines and lactone derivatives; these lactone derivatives were prepared by the oxidation of free saccharides using iodine in methanol at elevated temperature. Kurth et al. directly synthesized styrene derivatives in organic solvent using cation exchange resin and vinylbenzyl oxime while heating (Fig. 2C) $(23,24)$; the reducing end of the resulting monomeric saccharide moieties formed ring-opening structures. Narumi et al. synthesized maltopentaosyl styrene monomers by using 4-aminoalkyl styrene in methanol (25). Glycomonomers were commonly synthesized from free saccharides via glycosylamines prepared using an excess amount of ammonium hydrogen carbonate in water. These glycosylamines are unstable and must be handled carefully; when reacted with a carboxylic acid chloride such as acryloyl chloride or methacryloyl chloride, or with isocyanates or epoxides, these glycosylamines give rise to the corresponding $N$-linked glycomonomers (26-30). Kobayashi et al. synthesized styrene derivatives possessing $\alpha 2-3$ and $\alpha 2-6$ linked sialyllactose by coupling glycosylamine and $p$-vinylbenzoyl chloride (Fig. 2D) (31). Wulff et al. synthesized a $\mathrm{C}-\mathrm{C}$ bond-linked glycomonomer using $\mathrm{N}, \mathrm{N}$-dimethylbarbituric acid and 4-vinylbenzyl bromide with a saccharide derivative containing a barbiturate ring at the anomeric position (Fig. 2E) (32). Enzymatic syntheses of glycomonomers using glycosidase or lipase have also been reported (Fig. 2F, G) $(33,34)$.

All the above methods can only be applied to linear and relatively low molecular weight saccharides such as mono-, di-, and trisaccharides because higher molecular weight oligosaccharides are poorly soluble in organic solvents and are susceptible to cleavage of the glycosyl linkage, resulting in low yields. Therefore, a simpler and more efficient synthetic method that is applicable to various free oligosaccharides is required for the preparation of glyco-monomers and -polymers. 
(A)

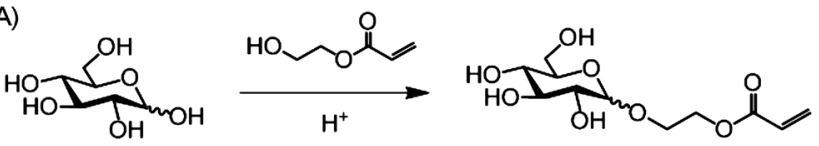

(B)
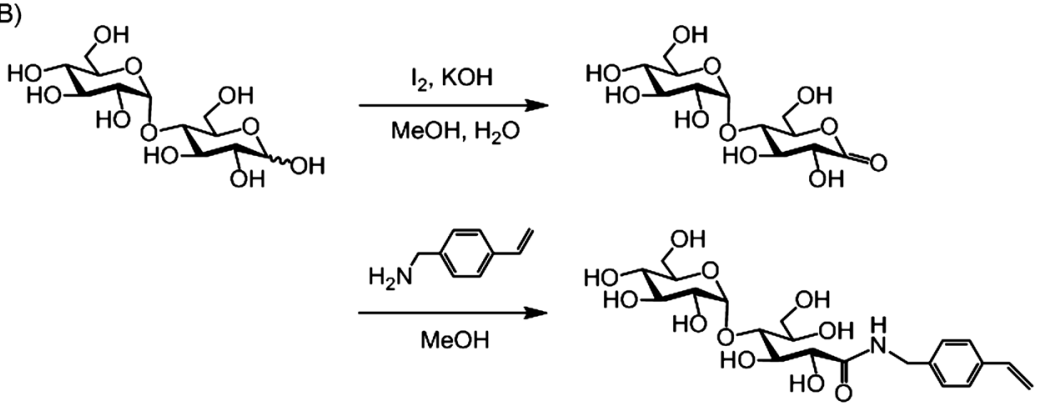

(C)

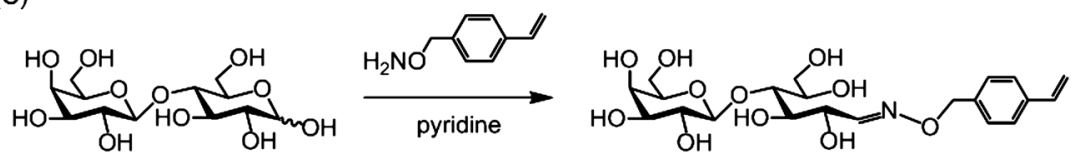

(D)
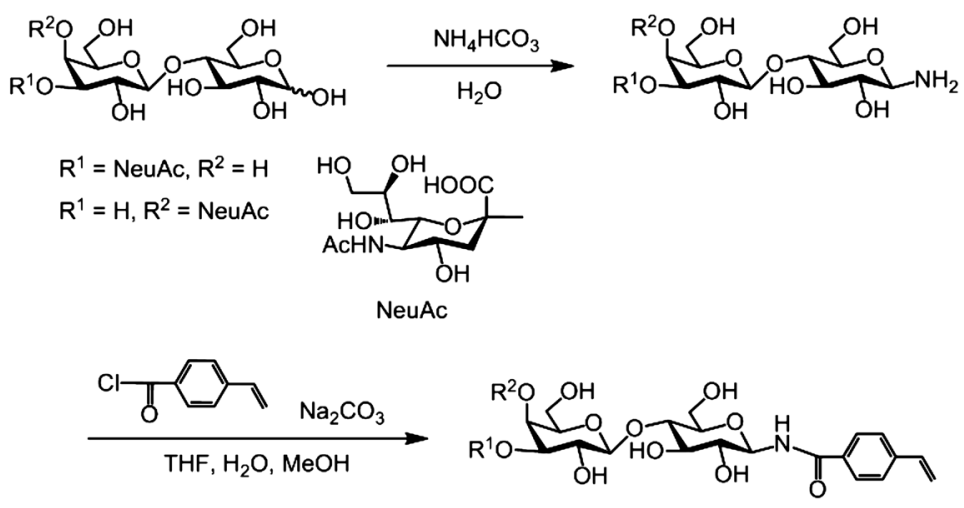

(E)

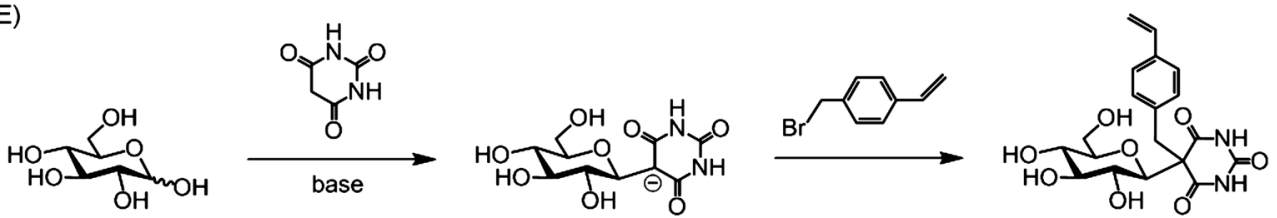

(F)

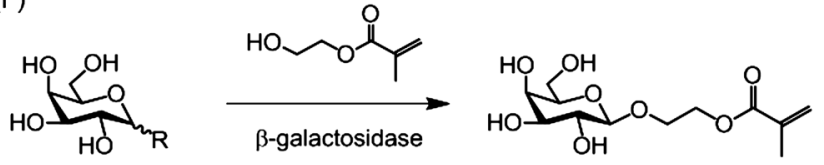

$\mathrm{R}=\mathrm{OH}, \beta-\mathrm{OEt}, \beta-\mathrm{OPr}$

(G)

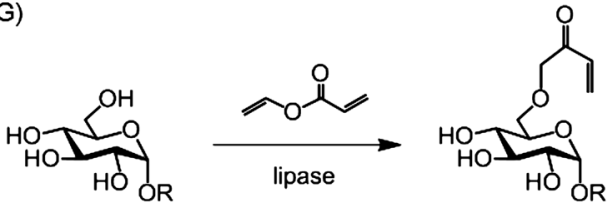

Fig. 2. Examples of protecting-group-free synthesis of glycomonomers from free saccharides. 


\section{Protecting-Group-Free Synthesis of Glycopolymers via Direct Anomeric Activation of Free Saccharides}

We recently developed an approach for activating the anomeric hydroxy group on free saccharides using a water-soluble dehydrative condensing agent, 2-chloro-1,3-dimethylimidazolinium chloride (DMC) (35-40). This method, called "Shoda activation" (41), does not require the use of any protecting group and the reaction can be conducted in water. The reactions proceed through a reactive intermediate formed as a result of preferential attack of the anomeric hydroxy group towards DMC, based on the fact that the $\mathrm{p} K_{\mathrm{a}}$ value of hemiacetal anomeric hydroxy group is much lower than that of other hydroxy groups on saccharide and water (42). The proposed reaction mechanism for DMC-mediated formation of $\beta$-glucoside is shown in Fig. 3. The first step is a nucleophilic attack of anomeric hydroxy group on $\beta$-glucose to the 2 position of DMC, giving rise to the $\beta$-glucosyl intermediate. The 2-hydroxy group of $\beta$-glucosyl intermediate then attacks the anomeric carbon atom to afford a 1,2-anhydro intermediate, which is selectively converted to the $\beta$-glucoside via intermolecular attack of the nucleophile. $\alpha$-Glucose that is in equilibrium with $\beta$-glucose also reacts with DMC to give the corresponding $\alpha$-glucosyl intermediate, which is converted to $\beta$-glucoside and regenerated to free saccharide by the attack of the nucleophile and water, respectively. In this section, the protecting-group-free synthesis of glycomonomers from free saccharides using DMC, and the subsequent polymerization reaction to obtain glycopolymers, are described below.

\section{C-1. Synthesis of Glycopolymers Bearing Triazole-} Linked Oligosaccharides Using Click Chemistry (43)

The triazole-linked glycomonomers $\mathbf{2}$ were synthesized from free saccharides in two steps without the use of any protecting group (Fig. 4). The first step is direct synthesis of $\beta$-glycosyl azides 1 using DMC and sodium azide under basic conditions in water (35). The resulting glycosyl azides were purified by ion-exchange column chromatography (44) or gel permeation chromatography. The second step is a copper(I)-catalyzed azide-alkyne cycloaddition reaction (CuAAC) between $\mathbf{1}$ and $N$-propargyl acrylamide in the presence of a catalytic amount of copper(II) sulfate pentahydrate, L-ascorbic acid sodium salt, and tris[(1-benzyl-1H-1,2,3triazol-4-yl)-methyl]amine (TBTA) used as a powerful stabilizing ligand for copper(I) in aqueous DMF (45). These reactants provide glycomonomers having the triazole-linked acrylamide group $\mathbf{2}$ in good yield. Click chemistry, such as CuAAC (46) and the thiol-ene reaction $(47,48)$, is also a powerful tool for synthesizing glycomonomers, glycopolymers, and other glycoclusters, as described in several reports on the application of click chemistry to glycotechnology (49-51). Our protecting-group-free method is applicable to not only disaccharides such as lactose (Lac), but also to higher molecular weight oligosaccharides having $\mathrm{N}$-acetylneuramic acid (NeuAc), including $\alpha 2-6$ sialyllactose (NeuAc $\alpha 2-6 \mathrm{Lac}$ ) and complex-type biantennary sialyl decasaccharide ( $N$-glycan).

The glycomonomers 2 were subjected to copolymerization with acrylamide (AAm) using a reversible addition-fragmentation chain transfer (RAFT) technique $(52,53)$ to obtain glycopolymers bearing triazole-linked oligosaccharides 3 . RAFT polymerization was performed in DMSO at $35^{\circ} \mathrm{C}$ using $2,2^{\prime}$-azobis(4methoxy-2,4-dimethylvaleronitrile) (V-70) and 2-(benzylsulfanylth iocarbonylsulfanyl)ethanol as the initiator and chain transfer agent (CTA), respectively. The glycopolymers 3 were obtained from free saccharides without the use of any protecting group. The saccharide unit ratios in the product polymers were found to be slightly

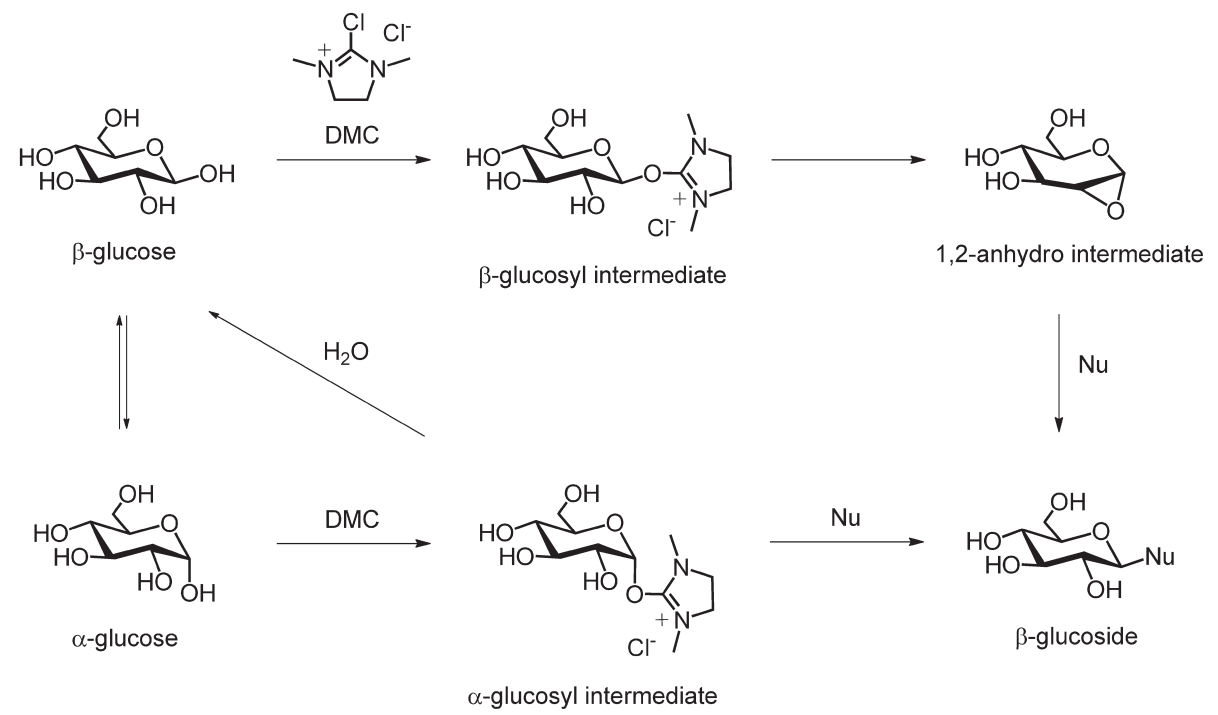

Fig. 3. Proposed reaction mechanism of direct $\beta$-glycoside formation from free saccharide. 


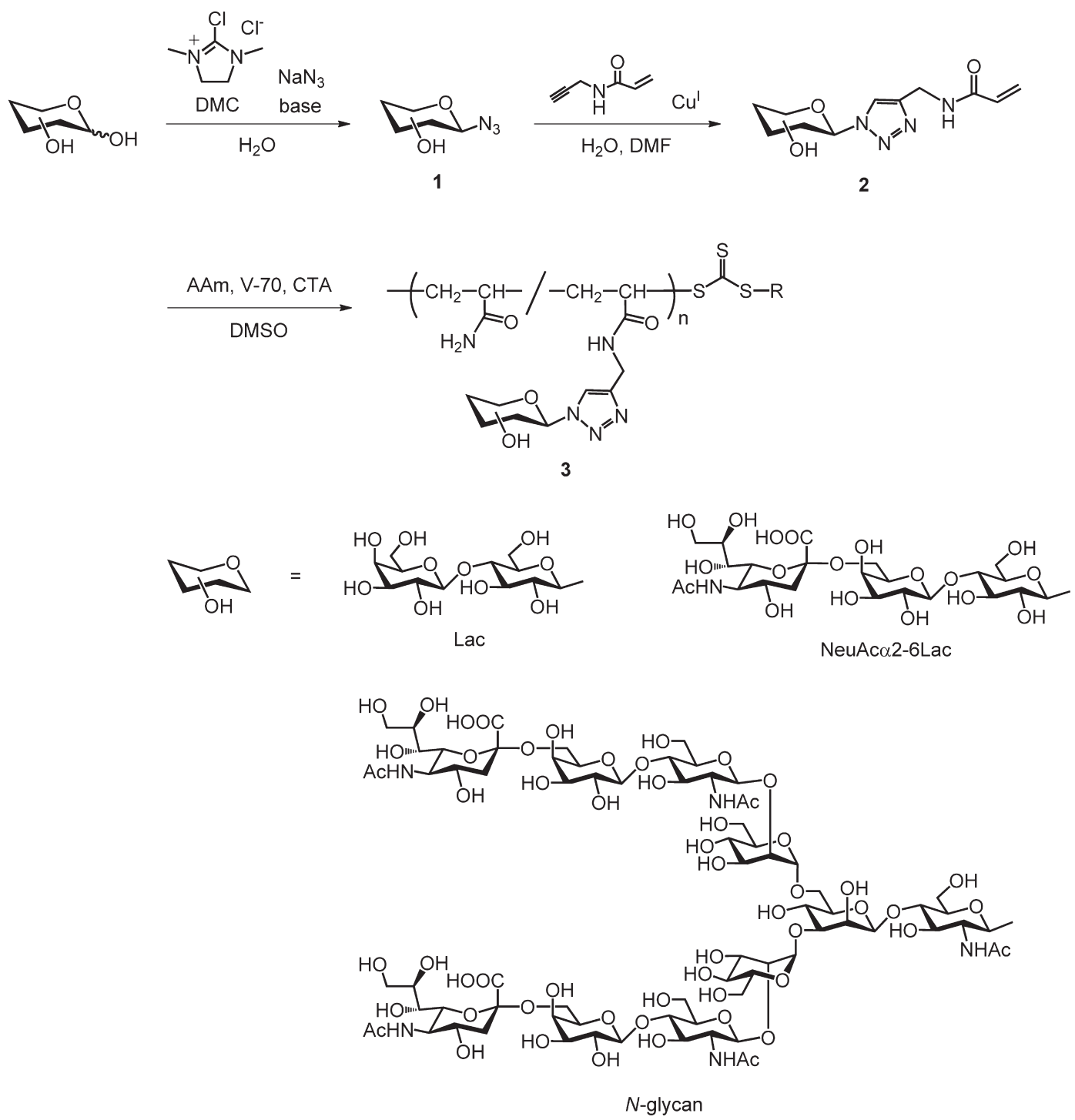

Fig. 4. Protecting-group-free synthesis of triazole-linked glycomonomers 2 via direct azidation and CuAAC, and subsequent polymerization to synthesize glycopolymers 3 .

lower (around 7\%) than the glycomonomer ratios in the feed (10\%). Lac-containing monomer provided 3 with low dispersity $\left(M_{\mathrm{w}} / M_{\mathrm{n}}=1.16\right)$, whereas $N$-glycan-containing monomers provided 3 with wide dispersity due to steric hindrance of the larger oligosaccharide. Although there have been many reports of the synthesis of glycopolymers, the above synthetic method is the first to incorporate a large biologically relevant oligosaccharide.

\section{C-2. Synthesis of Glycopolymers Bearing Thioglyco- sides via One-Pot Monomer Synthesis (54)}

The thioglycoside monomers $\mathbf{5}$ were synthesized from free saccharides by a one-pot method via 4-aminophenyl-1-thioglycosides (Fig. 5). The first step is direct synthesis of thioglycoside using DMC and 4-aminobenzenthiol under basic conditions in aqueous acetonitrile solution (38). D-Mannose (Man) and Lac were preferentially converted to $\alpha$ - and $\beta$-thioglycosides 4 , respectively. A hydroxy group at the 2-position of the saccharide strongly affects the anomeric structure of the product because one of the intermediates in this reaction is 1,2-anhydro sugar as shown in Fig. 3 (37). The second step is coupling with acryloyl chloride. After the first step reaction, the reaction mixture was washed with chloroform to thoroughly extract excess 4-aminobenzentiol, then the mixture was directly reacted with acryloyl chloride in the presence of triethylamine in aqueous THF solution to yield thioglycoside monomers 5 without isolation of 4 . Removal by preparative HPLC of the small amount of stereoisomeric by-product provided the glycomonomers in good yield.

The glycomonomers 5 were subjected to RAFT copolymerization with AAm to obtain glycopolymers bearing thioglycosides 


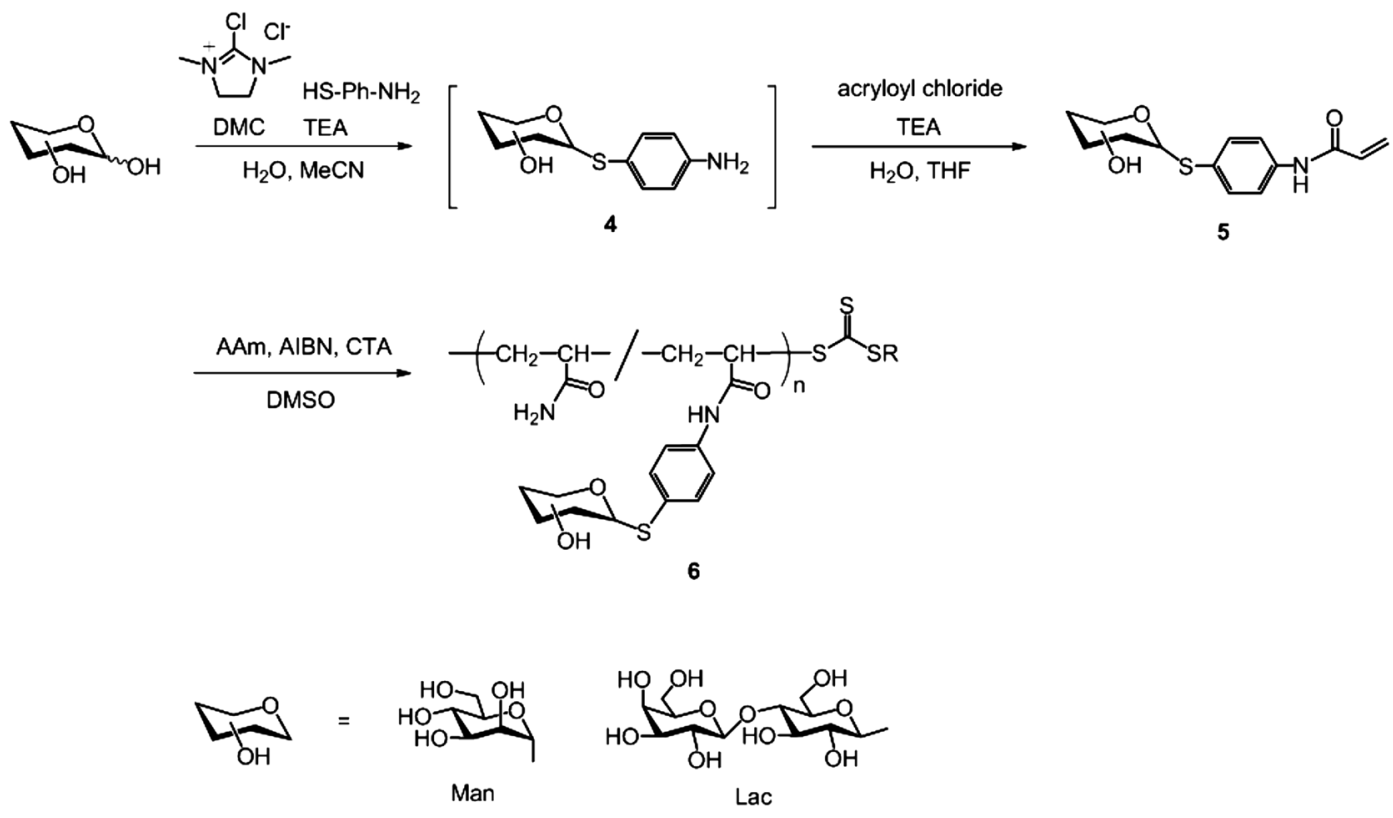

Fig. 5. One-pot synthesis of thio-linked glycomonomers 5 and subsequent polymerization to synthesize glycopolymers 6.

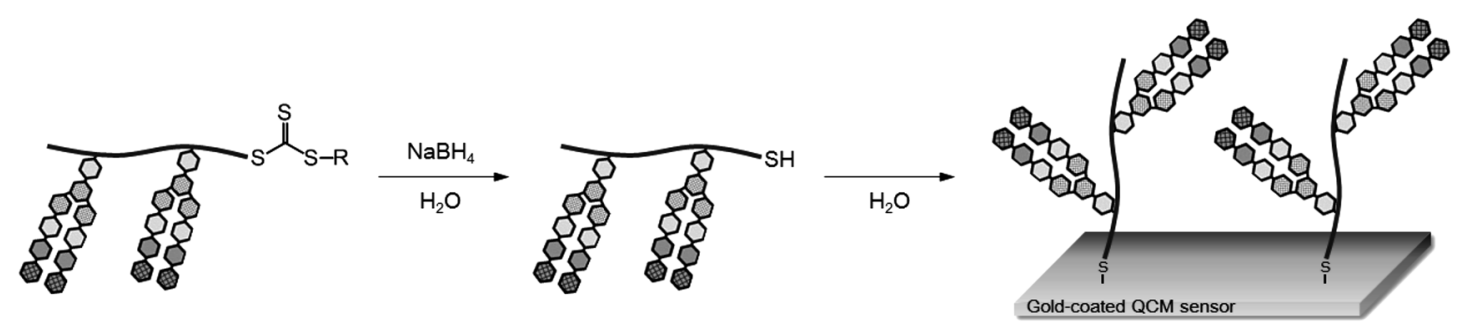

Fig. 6. Immobilization of glycopolymers on a gold-coated QCM sensor.

6. The polymerization reaction was performed in DMSO at $70^{\circ} \mathrm{C}$ using $\alpha, \alpha^{\prime}$-azobisisobutyronitrile (AIBN) and CTA. The glycopolymers 6 were obtained from free saccharides using a one-pot monomer synthesis method from free saccharides without the use of any protecting group. The glycopolymers 6 were found to have low dispersity $\left(M_{\mathrm{w}} / M_{\mathrm{n}}<1.3\right)$ and the saccharide unit ratios in the product were consistent with the monomer ratios in the feed. This one-pot approach from free saccharides is facile and effective for the synthesis of glycomonomers.

\section{Binding Assay of Glycopolymers}

The interactions of the resulting glycopolymers with lectins and influenza viruses were analyzed using the quartz crystal microbalance (QCM) method and hemagglutination inhibition (HI) assay, respectively, as described below.

\section{D-1. QCM Binding Analysis of Glycopolymers with Lectins}

The QCM method allows detection of nanogram increases in mass and is used to analyze the interaction of various molecules, including saccharide-protein complexes (55). The glycopolymers 3 and $\mathbf{6}$ synthesized by the RAFT polymerization method possess a trithiocarbonate group, resulting from the CTA, at the terminus of the polymer backbone; consequently, $\mathbf{3}$ and $\mathbf{6}$ were easily converted to thiol-terminated polymers in the presence of sodium borohydride (Fig. 6). The thiol-terminated glycopolymers were immobilized on a gold-coated QCM sensor via $\mathrm{Au}-\mathrm{S}$ bond formation, then subjected to a lectin binding test in phosphate buffered saline (PBS). Both triazole- and thio-linked glycopolymers strongly bound with the corresponding lectins: Lac with peanut lectin from Arachis hypogaea (PNA), NeuAc $\alpha 2-6$ Lac with Sambucus sieboldiana agglutinin (SSA), $N$-glycan with SSA, and Man with Concanavalin A from Canavalia ensiformis (ConA). Bovine serum albumin (BSA) did not interact with the glycopolymers. The association constants $\left(K_{\mathrm{a}}\right)$ for the lectin-saccharide interactions were estimated based on equilibrium frequency shifts on the order of $10^{7} \mathrm{M}^{-1}$. The $K_{\mathrm{a}}$ value for the binding of lectin and free saccharide is on the order of $10^{3} \mathrm{M}^{-1}(56)$. These results clearly indicate that the glycopolymers strongly interact with the corresponding lectin without nonspecific adsorption, 
Table 1. HI activities of triazole-linked glycopolymers 3 against human and avian influenza viruses.

\begin{tabular}{lcc}
\hline \multicolumn{1}{c}{ Glycopolymer $\mathbf{3}$} & $\begin{array}{c}\text { Human influenza } \\
\text { virus }^{\mathrm{a}}\end{array}$ & $\begin{array}{c}\text { Avian influenza } \\
\text { virus }^{\mathrm{b}}\end{array}$ \\
\hline Lac-trz-PAAm & $\mathrm{ND}^{\mathrm{c}}$ & $\mathrm{ND}$ \\
NeuAc $\alpha$ 2-6Lac-trz-PAAm & $2.5 \times 10^{-4}$ & $\mathrm{ND}$ \\
$N$-Glycan-trz-PAAm & $4.9 \times 10^{-7}$ & $6.3 \times 10^{-5}$ \\
Fetuin & $7.8 \times 10^{-5}$ & $5.0 \times 10^{-3}$ \\
\hline
\end{tabular}

${ }^{\mathrm{a}}$ The minimum concentration $(\mathrm{g} / \mathrm{mL})$ for binding human influenza virus A/Memphis/1/1971 (H3N2). ${ }^{\mathrm{b}}$ The minimum concentration $(\mathrm{g} / \mathrm{mL})$ for binding avian influenza virus A/duck/Hong Kong/313/4/1978 (H5N3).

${ }^{\mathrm{c}}$ Not detected (no activity).

suggesting that the glycopolymers exhibit the glycocluster effect.

\section{D-2. HI Assay of Glycopolymers against Influenza} Viruses

Viral hemagglutinin on the influenza virus is known to bind with sialylsaccharides. Binding of the triazole-linked glycopolymers 3 with influenza viruses was demonstrated using the HI assay (Table 1). Triazole-linked NeuAc $\alpha 2-6$ Lac-bearing glycopolymer (NeuAc $\alpha 2-6$ Lac-trz-PAAm; minimum concentration $2.5 \times 10^{-4} \mathrm{~g} /$ $\mathrm{mL}$ ) and $N$-glycan-bearing glycopolymer ( $N$-glycan-trz-PAAm; minimum concentration $4.9 \times 10^{-7} \mathrm{~g} / \mathrm{mL}$ ) bound to human influenza virus A/Memphis/1/1971 (H3N2). No activity was observed with triazole-linked Lac-bearing glycopolymer (Lac-trz-PAAm) lacking a NeuAc residue. A minimum concentration of $7.8 \times 10^{-5} \mathrm{~g} / \mathrm{mL}$ fetuin, a blood protein bearing sialyloligosaccharides, was required to obtain a positive HI result. $N$-glycan-trz-PAAm bound human influenza virus, recognized by the $\alpha 2-6$ sialylgalactosyl residue, 510- and 160-fold more strongly than NeuAc $\alpha 2-6$ Lac-trz-PAAm and fetuin, respectively. Interestingly, Lac-trz-PAAm and NeuAc $\alpha 2-6$ Lac-trz-PAAm exhibited no activity towards avian influenza virus A/duck/Hong Kong/313/4/1978 (H5N3), which is primarily recognized by the $\alpha 2-3$ sialylgalactosyl residue, whereas $N$-glycantrz-PAAm, which possesses only an $\alpha 2-6$ sialylgalactosyl residue, did exhibit activity against avian influenza virus at a minimum concentration of $6.3 \times 10^{-5} \mathrm{~g} / \mathrm{mL}$ glycopolymer. This binding is 80 times stronger than that of fetuin. These results indicate that $\mathrm{N}$ glycan-trz-PAAm binds much more strongly with both human and avian influenza viruses than NeuAc $\alpha 2-6$ Lac-trz-PAAm, suggesting that the natural structure of oligosaccharides is important for interaction with biological receptors such as those in influenza viruses.

\section{E. Conclusion}

This article reviewed several methods, including our own recent findings, for the protecting-group-free synthesis of glycomonomers and -polymers from free saccharides. Simple and efficient synthetic approaches for generating glycopolymers from free saccharides that are applicable to various higher molecular weight oligosaccharides, such as $\mathrm{N}$-linked and $\mathrm{O}$-linked oligosaccharides typically found on cell surfaces, are required to advance glycotechnology. Our synthetic method, utilizing direct azidation and CuAAC, generated $N$-glycan-bearing glycopolymers from free saccharides without the use of any protecting group. The results of assays using these glycopolymers suggest that multivalency and the natural structure of the oligosaccharide amplify saccharide-protein interactions. In the near future, glycopolymers will likely play significant roles in novel biomaterials such as pathogen inhibitors, cell cultivation agents for tissue engineering, and biosensors for the detection of viruses and toxins.

\section{Acknowledgments}

The author thanks Prof. Shin-ichiro Shoda (Tohoku University), Prof. Yoshiharu Kimura (Kyoto Institute of Technology), Prof. Yoshiko Miura (Kyushu University), and all of collaborators for their continuous encouragement and discussions throughout this study. The author also thanks Prof. Takashi Suzuki and Assoc. Prof. Tadanobu Takahashi (University of Shizuoka) for conducting the HI assays, and acknowledges the gift of $N$-glycan from Prof. Kenji Yamamoto (Ishikawa Prefectural University) and Prof. Hisashi Ashida (Kinki University). This work was financially supported by JSPS KAKENHI Grant No. 25810075.

\section{References}

1. Narain, R. (2011) Engineered Carbohydrate-Based Materials for Biomedical Applications, John Wiley \& Sons, Inc.

2. Droumaguet, L. B., and Nicolas, J. (2010) Polym. Chem. 1, 563-598.

3. Slavin, S., Burns, J., Haddleton, D. M., and Becer, C. R. (2011) Eur. Polym. J. 47, 435-446.

4. Miura, Y. (2012) Polym. J. 44, 679-689.

5. Sunasee, R., and Narain, R. (2012) Macromol. Biosci. 13, 9-27.

6. Ahmed, M., and Narain, R. (2013) Eur. Polym. J. 49, 3010-3033.

7. Lee, Y. C., and Lee, R. T. (1995) Acc. Chem. Res. 28, 321-327.

8. Mammen, M., Choi, S. K., and Whitesides, G. M. (1998) Angew. Chem. Int. Ed. 37, 2755-2794.

9. Woller, E. K., and Cloninger, M. J. (2002) Org. Lett. 4, 7-10.

10. Chabre, Y. M., and Roy, R. (2008) Curr. Top. Med. Chem. 8, 1237-1285.

11. Tanaka, K., Siwu, E. R. O., Minami, K., Hasegawa, K., Nozaki, S., Kanayama, Y., Koyama, K., Chen, W. C., Paulson, J. C., Watanabe, Y., and 
Fukase, K. (2010) Angew. Chem. Int. Ed. 49, 8195-8200.

12. de la Fuente, J. M., Barrientos, A. G., Rojas, T. C., Rojo, J., Canada, J., Fernandez, A., and Penades, S. (2010) Angew. Chem. Int. Ed. 40, $2258-2261$.

13. de la Fuente, J. M., and Penades, S. (2005) Tetrahedron Asymmetry 16, 387-391.

14. Spain, S. G., Albertin, L., and Cameron, N. R. (2006) Chem. Commun. (Camb.), 4198-4200.

15. Housni, A., Cai, H., Liu, S., Pun, S. H., and Narain, R. (2007) Langmuir 23, 5056-5061.

16. Ejaz, M., Ohno, K., Tsujii, Y., and Fukuda, T. (2000) Macromolecules 33, 2870-2874.

17. Ynag, Q., Xu, Z. K., Hu, M. X., Li, J. J., and Wu, J. (2005) Langmuir 21, 10717-10723.

18. Geng, J., Mantovani, G., Tao, L., Nicolas, J., Chen, G. J., Wallis, R., Mitchell, D. A., Johnson, B. R. G., Evans, S. D., and Haddleton, D. M. (2007) J. Am. Chem. Soc. 129, 15156-15163.

19. Guo, T. Y., Liu, P., Zhu, J. W., Song, M. D., and Zhang, B. H. (2006) Biomacromolecules 7, 1196-1202.

20. Kitazawa, S., Okumura, M., Kinomura, K., and Sakakibara, T. (1990) Chem. Lett. 9, 1733-1736.

21. Kobayashi, K., Sumitomo, H., and Ina, Y. (1985) Polym. J. 17, 567-575.

22. Narain, R., and Armes, S. P. (2003) Biomacromolecules 4, 1746-1758.

23. Zhou, W.-J., Wilson, M. E., Kurth, M. J., Hsieh, Y.-L., Krochta, J. M., and Shoemaker, C. F. (1997) Macromolecules 30, $7063-7068$.

24. Zhou, W.-J., Naik, S. S., Kurth, M. J., Hsieh, Y.-L., and Krochta, J. M. (1998) J. Polym. Sci. A Polym. Chem. 36, $2971-2978$.

25. Togashi, D., Otsuka, I., Borsali, R., Narumi, A., and Kawaguchi, S. (2015) J. Polym. Sci. A Polym. Chem. 53, 1671-1679.

26. Whistler, R. L., and Goatley, J. L. (1961) J. Polym. Sci., Polym. Phys. Ed. 50, 127-132.

27. Whistler, R. L., Panzer, H. P., and Roberts, H. J. (1961) J. Org. Chem. 26, 1583-1588.

28. Klein, J., and Herzog, D. (1987) Macromol. Chem. Phys. 188, 1217-1232.

29. Klein, J., and Begli, A. H. (1989) Macromol. Chem. Phys. 190, 2527-2534.

30. Klein, J., Begli, A. H., and Engelke, S. (1989) Makromol. Chem., Rapid. Commun. 10, 629-636.

31. Tsuchida, A., Kobayashi, K., Matsubara, N., Muramatsu, T., Suzuki, T., and Suzuki, Y. (1998) Glycoconj. J. 15, $1047-1054$.

32. Wulff, G., and Clarkson, G. (1994) Macromol. Chem. Phys. 195, 2603-2610.

33. Gill, I., and Valivety, R. (2000) Angew. Chem. Int. Ed. 39, 3804-3808.

34. Degoede, A., Vanoosterom, M., Vandeurzen, M. P. J., Sheldon, R. A., Vanbekkum, H., and Vanrantwijk, F. (1993) Heterogeneous Catalysis and Fine Chemicals III, Eds. Guisnet, M., Barbier, J., Barranult, J., Bouchoule, C., Duprez, D., Perot, G., and Montassier, C., Elsevier Science Vol.78, pp. 513-520.

35. Tanaka, T., Nagai, H., Noguchi, M., Kobayashi, A., and Shoda, S. (2009) Chem. Commun. (Camb.), 3378-3379.

36. Noguchi, M., Tanaka, T., Gyakushi, H., Kobayashi, A., and Shoda, S. (2009) J. Org. Chem. 74, 2210-2212.

37. Tanaka, T., Huang, W. C., Noguchi, M., Kobayashi, A., and Shoda, S. (2009) Tetrahedron Lett. 50, 2154-2157.

38. Tanaka, T., Matsumoto, T., Noguchi, M., Kobayashi, A., and Shoda, S. (2009) Chem. Lett. 38, 458-459.

39. Yoshida, N., Noguchi, M., Tanaka, T., Matsumoto, T., Aida, N., Ishihara, M., Kobayashi, A., and Shoda, S. (2011) Chem. Asian J. 6, $1876-1885$.

40. Yoshida, N., Fujieda, T., Kobayashi, A., Ishihara, M., Noguchi, M., and Shoda, S. (2013) Chem. Lett. 42, 1038-1039.

41. Novoa, A., Barluenga, S., Serba, C., and Winssinger, N. (2013) Chem. Commun. (Camb.) 49, 7608-7610.

42. Thamsen, J., Lund, E. W., Åkerström, Å., Finsnes, E., Sörensen, J. S., and Sörensen, N. A. (1952) Acta Chem. Scand. 6, $270-284$.

43. Tanaka, T., Ishitani, H., Miura, Y., Oishi, K., Takahashi, T., Suzuki, T., Shoda, S., and Kimura, Y. (2014) ACS Macro Lett. 3, $1074-1078$.

44. Vinson, N., Gou, Y., Becer, C. R., Haddleton, D. M., and Gibson, M. I. (2011) Polym. Chem. 2, 107-113.

45. Chan, T. R., Hilgraf, R., Sharpless, K. B., and Fokin, V. V. (2004) Org. Lett. 6, 2853-2855.

46. Kolb, H., Finn, M., and Sharpless, K. (2001) Angew. Chem. Int. Ed. 40, 2004-2021.

47. Chen, G. J., Amajjahe, S., and Stenzel, M. H. (2009) Chem. Commun. (Camb.), 1198-1200.

48. You, L. C., and Schlaad, H. (2006) J. Am. Chem. Soc. 128, 13336-13337.

49. Witczak, Z. J., and Bielski, R. (2013) Click Chemistry in Glycoscience, John Wiley \& Sons, Inc.

50. Dondoni, A. (2007) Chem. Asian J. 2, 700-708.

51. Binder, W., and Sachsenhofer, R. (2007) Macromol. Rapid Commun. 28, $15-54$.

52. Moad, G., Rizzardo, E., and Thang, S. H. (2005) Aust. J. Chem. 58, 379-410.

53. Lowe, A. B., Sumerlin, B. S., and McCormick, C. L. (2003) Polymer (Guildf.) 44, 6761-6765.

54. Tanaka, T., Inoue, G., Shoda, S., and Kimura, Y. (2014) J. Polym. Sci. A Polym. Chem. 52, 3513-3520.

55. Ebara, Y., and Okahata, Y. (1994) J. Am. Chem. Soc. 116, 11209-11212.

56. Miura, Y., Ikeda, T., and Kobayashi, K. (2003) Biomacromolecules 4, 410-415.

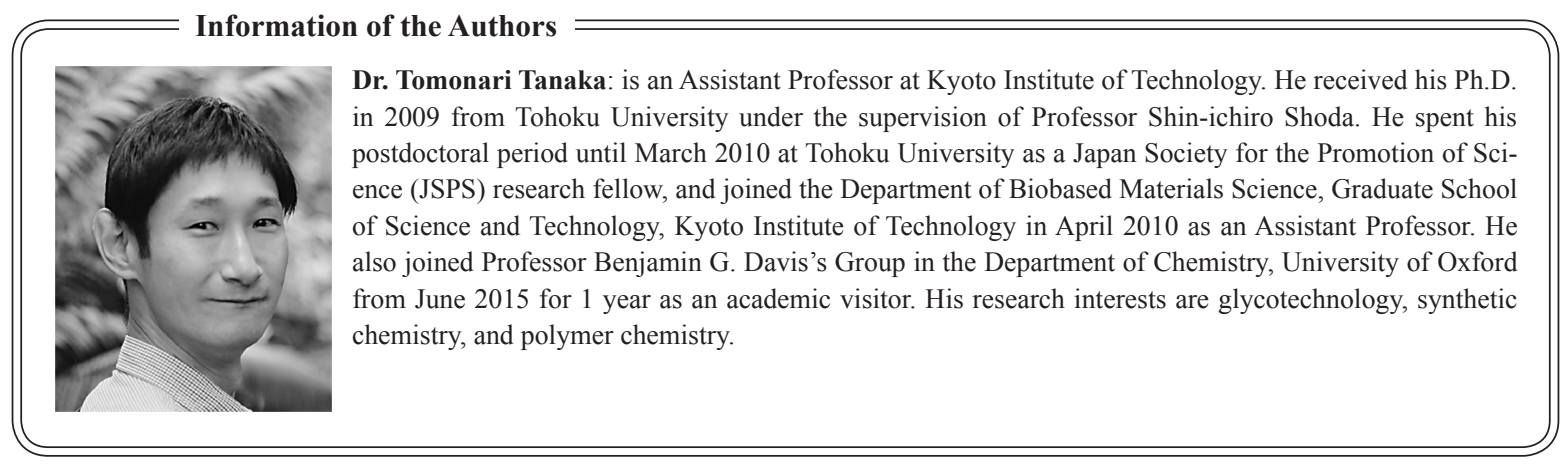

